\title{
Transflective In-Plane Switching Liquid Crystal Display
}

\author{
Ruibo Lu, Zhibing Ge, Qi Hong, and Shin-Tson Wu, Fellow, IEEE
}

\begin{abstract}
A single cell gap, single gamma curve, and low operating voltage transflective liquid crystal display (LCD) using an in-plane switching (IPS) cell is proposed. In the IPS cell, the pixilated transparent indium tin oxide electrodes are on the top substrate and the reflectors are on the bottom substrate. The electrooptic properties of the transflective IPS LCD are calculated using three-dimensional simulation software. By optimizing the reflector width, the voltage-dependent transmittance and reflectance curves can be matched.
\end{abstract}

Index Terms-Color, in-plane switching, response time, transflective liquid crystal display.

\section{INTRODUCTION}

$\mathbf{T}$ RANSFLECTIVE liquid crystal displays (LCDs) have been widely used in portable displays such as cell phones, electronic books, and personal computers because its readability is not limited by the ambient lighting conditions. Quite a few liquid crystal (LC) modes have been proposed for transflective LCDs, e.g., the mixed-mode twisted nematic (MTN) mode [1], homogeneous mode [2], optically compensated bend (OCB) mode [3], vertical aligned (VA) mode [4], [5], hybrid-aligned nematic (HAN) mode [6], in-plane switching (IPS) mode [7]-[9] and fringe-field switching (FFS) mode [10], [11]. The cell configurations can be divided into double cell gap or single cell gap, and single thin-film transistor (TFT) or double TFT driving circuits. A detailed review can be found in [12]. At present, the mainstream transflective LCDs uses the double cell gap approach for two reasons: 1) both transmissive (T) and reflective $(\mathrm{R})$ modes can achieve maximum light efficiency and 2) the gamma curves between the T-and R-modes match almost perfectly. However, two shortcomings are inherent: 1) the T-mode has a slower response time than the R-mode because its cell gap is $\sim 2 \times$ thicker than that of the R-mode and 2) the viewing angle is relatively narrow, especially when the homogeneous cells are employed.

For practical applications, a single cell gap, single gamma curve, single TFT per pixel, and wide view transflective LCD is desired. IPS mode is known to exhibits wide viewing angle because of its in-plane switching mechanism. Several approaches using IPS mode for transflective LCDs have been explored. For

Manuscript received November 5, 2006; revised December 6, 2006. This work was supported Toppoly Optoelectronics Corporation (Taiwan).

The authors are with the College of Optics and Photonics, University of Central Florida, Orlando, FL 32816 USA (e-mail: rlu@mail.ucf.edu).

Color versions of one or more of the figures are available online at http:// ieeexplore.ieee.org.

Digital Object Identifier 10.1109/JDT.2006.890696 instance, Song et al. [7] proposed a single cell gap and single TFT transflective IPS-LCD using the interdigitated reflective electrodes on the bottom substrate. The major advantage of this approach is simple fabrication process. However, two tradeoffs are found: a relatively high operating voltage $\left(\sim 7 V_{\text {rms }}\right)$ and mismatched voltage dependent transmittance (VT) and reflectance (VR) curves [also called gamma curves]. In [7], the in-cell phase retarder is laid on top of the reflective electrodes. This in-cell polymeric film shields some of the electric field strength and results in an increased operating voltage. Another transflective IPS-LCD using zigzag electrodes was proposed by Park et al. [8], where the electrodes are divided into two different transmissive and reflective regions. To realize a single cell gap and single gamma curve, the electrode gap and the angle between LC directors and the electrodes at the different parts need to be optimized [9]. In addition to the complicated electrode structure, the required operating voltage is still relatively $\operatorname{high}\left(\sim 7 V_{\mathrm{rms}}\right)$.

To overcome the abovementioned problems, in this paper we present a transflective IPS LCD with separated electrodes and reflectors, where the pixilated transparent indium-tin-oxide (ITO) electrodes are on the top substrate and the bumpy reflectors on the bottom substrate. The fringing electric field near the top substrate is not affected by the bottom in-cell phase retarder. Thus, a low operating voltage is obtained. Moreover, by optimizing the reflector width, a matched gamma curve between VT and VR is achieved. Therefore, a single cell gap, single TFT, and single gamma curve transflective IPS LCD can be realized.

\section{Device Structure AND Working PrinciPLe}

Fig. 1 shows the device structure of the transflective IPS LCD. The interdigitated pixel and common electrodes are on the inner side of the top substrate, and the pixilated bumpy reflectors which align with the top electrodes are on the inner side of the bottom substrate. Since the top ITO electrodes are transparent, the regions between the neighboring reflectors are used as T-mode while the regions above the reflectors are used as R-mode. For simulations, we chose the electrode width to be $\sim 7 \mu \mathrm{m}$, electrode gap $\sim 10 \mu \mathrm{m}$, the width of the bumpy aluminum reflector $\sim 7 \mu \mathrm{m}$, cell gap $\sim 4 \mu \mathrm{m}$, and a Merck positive dielectric anisotropy $(\Delta \varepsilon)$ LC mixture MLC-6692.

Fig. 1 shows the working principles in the (a) voltage-off and (b) voltage-on states. At $V=0$, the LC directors are homogenously aligned in the $\mathrm{LC}$ cell with a pretilt angle $\theta \sim 2^{\circ}$, and the rubbing angle with respect to the stripe electrodes is $\phi=10^{\circ}$. The $\mathrm{LC}$ rubbing direction is arranged to be along the transmission axis of the linear polarizer LP1. Two broadband $\lambda / 4$ films 


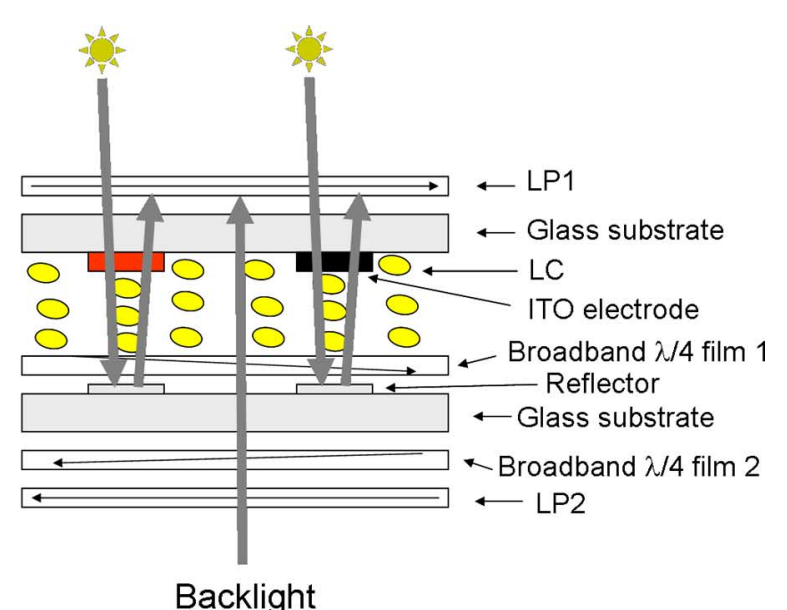

(a)

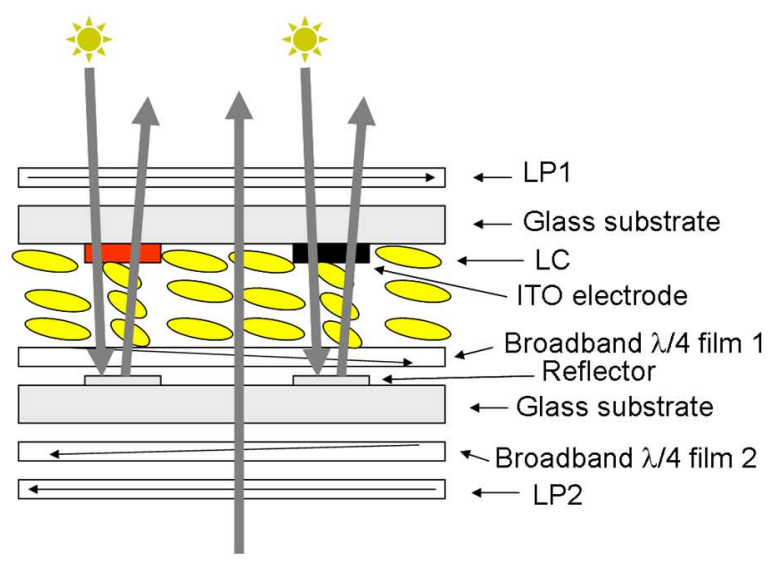

Backlight

(b)

Fig. 1. The device structure of the transflective IPS LCD and its working principles in the (a) voltage-off state and (b) voltage-on state.

consisting of a half-wave film and a quarter-wave film are used. As shown in Fig. 1, film 1 is imbedded between the LC layer and the reflectors as the in-cell phase retarder, and film 2 is laminated to the inner side of the bottom polarizer (LP2) whose transmittance axis is orthogonal to that of LP1. Although the fabrication of in-cell phase retarder is challenging, several groups [13], [14] have demonstrated its feasibility.

In the reflective mode, the incident linearly polarized ambient light from LP1 passes through the LC layer without changing its polarization, but becomes circularly polarized after propagating through the broadband $\lambda / 4$ film- 1 . Upon reflection, the light enters the broadband $\lambda / 4$ film-1 and the LC layer again and becomes a linearly polarized light with its polarization axis rotated by $90^{\circ}$. Therefore, it is absorbed by LP1 and the cell appears dark. In the T-mode, the incident light from the backlight unit passes through the linear polarizer LP2, broadband $\lambda / 4$ film-2, broadband $\lambda / 4$ film-1 (whose optic axis is orthogonal to that of the broadband $\lambda / 4$ film-2), and the LC layer, and is blocked by the second linear polarizer LP1. Both R- and T-modes are normally black.

When the applied voltage exceeds the Freederisckz transition threshold [Fig. 1(b)], the LC directors are twisted by the fringing electric field. Therefore, the light transmits through the transflective IPS device, and a bright state is obtained in both T- and $\mathrm{R}$-modes. To achieve maximum transmittance and reflectance simultaneously, the effective LC cell retardation value $(\mathrm{d} \cdot \Delta \mathrm{n})$ should be equal to $\lambda / 2$ for both $\mathrm{T}$-mode and R-mode.

\section{Simulation ApPROACHES AND Optical CAlCUlations}

To simulate the device performance, we first calculate the dynamic 3-D LC director distributions and then obtain the detailed electro-optical properties. We have developed a 3-D simulator for calculating the LC director distributions. Our 3-D simulator combines the finite element method and finite difference method in order to improve the calculation speed [15]. Once the LC director distribution profiles are obtained, we then calculate the electro-optical properties of the LCD at normal incidence using the extended Jones matrix method [16]. The LC layer is modeled as a stack of uniaxial homogeneous layers. Here, we assume the reflections between the interfaces are negligible. Therefore, the transmitted tangential electric fields $\left[\mathrm{E}_{\mathrm{x}}^{\text {exit }}\right.$ air, $\left.\mathrm{E}_{\mathrm{y}, \text { air }}^{\text {exit }}\right]$ are correlated to the incident tangential ones $\left[\mathrm{E}_{\mathrm{x}, \text { air }}^{\text {inc }}, \mathrm{E}_{\mathrm{y}, \text { air }}^{\text {inc }}\right]$ by

$$
\left[\begin{array}{c}
E_{x, \text { air }}^{\text {exit }} \\
E_{y, \text { air }}^{\text {exit }}
\end{array}\right]=\mathbf{J}\left[\begin{array}{c}
E_{x, \text { air }}^{\text {inc }} \\
E_{y, \text { air }}^{\text {inc }}
\end{array}\right]=\mathbf{J}_{\text {ext }} \mathbf{J}_{N} \mathbf{J}_{N-1} \cdots \mathbf{J}_{2} \mathbf{J}_{1} \mathbf{J}_{\text {ent }}\left[\begin{array}{c}
E_{x, \text { air }}^{\text {inc }} \\
E_{y, \text { air }}^{\text {inc }}
\end{array}\right]
$$

where $\mathbf{J}_{\text {ext }}$ and $\mathbf{J}_{\text {ent }}$ are the correction matrices considering the transmission losses in the air-LCD interface, which are given by

$$
\begin{aligned}
& \mathbf{J}_{\text {ent }}=\left[\begin{array}{cc}
\frac{2 \cos \theta_{p}}{\cos \theta_{p}+n_{p} \cos \theta_{k}} & 0 \\
0 & \frac{2 \cos \theta_{k}}{\cos \theta_{k}+n_{p} \cos \theta_{p}}
\end{array}\right] \\
& \mathbf{J}_{\text {ext }}=\left[\begin{array}{cc}
\frac{2 n_{p} \cos \theta_{k}}{\cos \theta_{p}+n_{p} \cos \theta_{k}} & 0 \\
0 & \frac{2 n_{p} \cos \theta_{p}}{\cos \theta_{k}+n_{p} \cos \theta_{p}}
\end{array}\right]
\end{aligned}
$$

where $\theta_{p}$ is given by

$$
\theta_{p}=\sin ^{-1}\left(\sin \left(\theta_{k}\right) / \operatorname{Re}\left(n_{p}\right)\right) .
$$

In (2) and (3), $n_{p}$ is the average refractive index of the polarizer $\left(n_{p}=\left(2 n_{e, p}+n_{o, p}\right) / 3\right), n_{e, p}=1.500+i \times 3.251 \times 10^{-3}$ and $n_{o, p}=1.500+i \times 2.86 \times 10^{-5}, \operatorname{Re}\left(n_{p}\right)$ stands for the real part of $n_{p}$, and $\theta_{k}$ is the azimuthal angle of the incident wavevector k.

To characterize the R-mode, we apply the mirror-image method [17] and the extended Jones matrix method to correlate the exit and incident electric fields [18]. As a result, the output transmittance or reflectance can be written as

$$
T(R)=\frac{\left|E_{x, \text { air }}^{\text {exit }} / \cos \theta_{k}\right|^{2}+\left|E_{y, \text { air }}^{\text {exit }}\right|^{2}}{\left|E_{x, \text { air }}^{\text {inc }} / \cos \theta_{k}\right|^{2}+\left|E_{y, \text { air }}^{\text {inc }}\right|^{2}} .
$$

Considering a randomly polarized light with equal perpendicular and parallel components, we can assume

$$
\left[\begin{array}{l}
E_{x, \text { air }}^{\text {inc }} \\
E_{y, \text { air }}^{\text {inc }}
\end{array}\right]=\left[\begin{array}{c}
\cos \theta_{k} \\
e^{i \varphi}
\end{array}\right]
$$




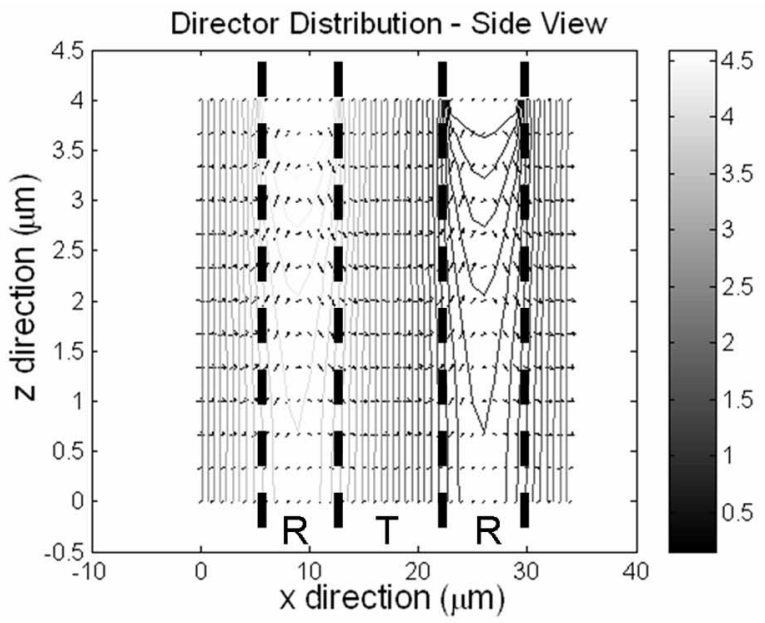

(a)

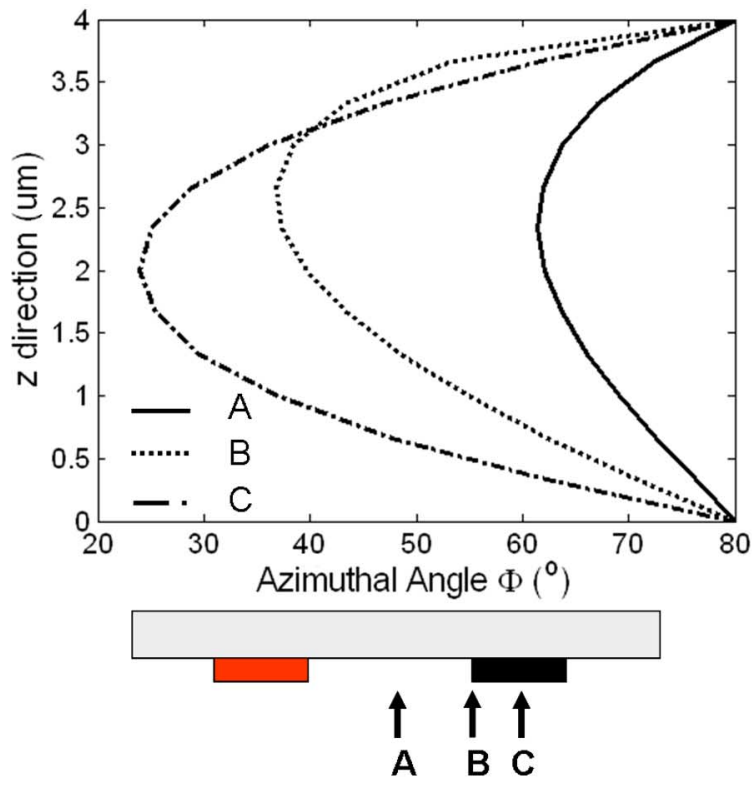

(b)

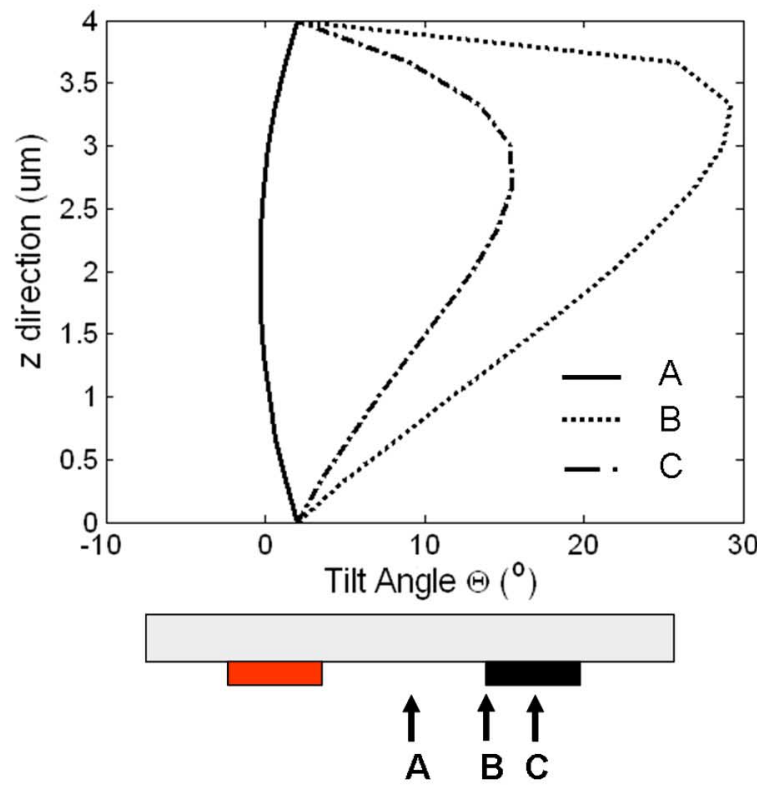

(c)

Fig. 2. Simulated (a) side view of LC director profiles of the IPS cell, and (b) azimuthal and (c) tilt angle at different positions along the $\mathrm{x}$-direction at $V=$ $4.75 V_{\text {rms }}$.

where $\varphi$ denotes the phase difference between the perpendicular and parallel components. From the extended Jones matrix correlating the exit and incident tangential fields, the exit components can be expressed as

$$
\begin{aligned}
& E_{x, \text { air }}^{e x i t}=J_{11} \cos \theta_{k}+J_{12} e^{j \varphi} \\
& E_{y, \text { air }}^{e x i t}=J_{21} \cos \theta_{k}+J_{22} e^{j \varphi} .
\end{aligned}
$$

Therefore, by substituting (6) and (7) into (4), we derive the averaged transmittance or reflectance as follows:

$$
\begin{aligned}
& T(R) \\
& \quad=\frac{\left|J_{11}\right|^{2} \cos ^{2} \theta_{k}+\left|J_{12}\right|^{2}+\cos ^{2} \theta_{k}\left(\left|J_{21}\right|^{2} \cos ^{2} \theta_{k}+\left|J_{22}\right|^{2}\right)}{2 \cos ^{2} \theta_{k}} .
\end{aligned}
$$

\section{Simulation Results AND Discussions}

\section{A. LC Director Distribution}

Fig. 2 plots the side view of the LC director distribution of the IPS LCD, and the corresponding azimuthal and tilt angle variations at different positions along the $\mathrm{x}$-direction at $V=$ $4.75 V_{\text {rms }}$. At the central position of the $\mathrm{T}$ region, A, the tilt angle remains almost unchanged while the azimuthal angle $\Phi$ is twisted $45^{\circ}$ on average from the initial state. However, at the electrode edge point, $\mathrm{B}$, in the R-region, the azimuthal angle is on the average of $32^{\circ}$ while the tilt angle reaches as high as $29^{\circ}$ near the electrode. It means the LC directors are not only twisted but also splayed. Similar situation is observed at point $\mathrm{C}$, where the azimuthal angle is averagely twisted at $10^{\circ}$ even though the maximum tilt angle is decreased by $15^{\circ}$. It can be concluded that the transverse electric fields between the neighboring electrodes twist the LC directors in the $\mathrm{x}-\mathrm{y}$ plane resulting in more phase retardation in the T-mode. Meanwhile, the R-part is only 


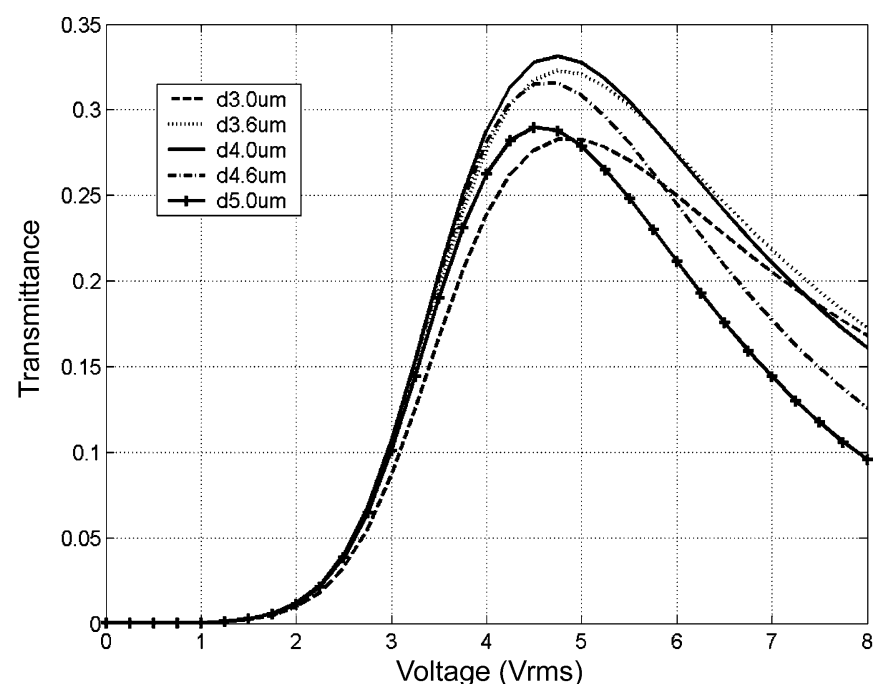

(a)

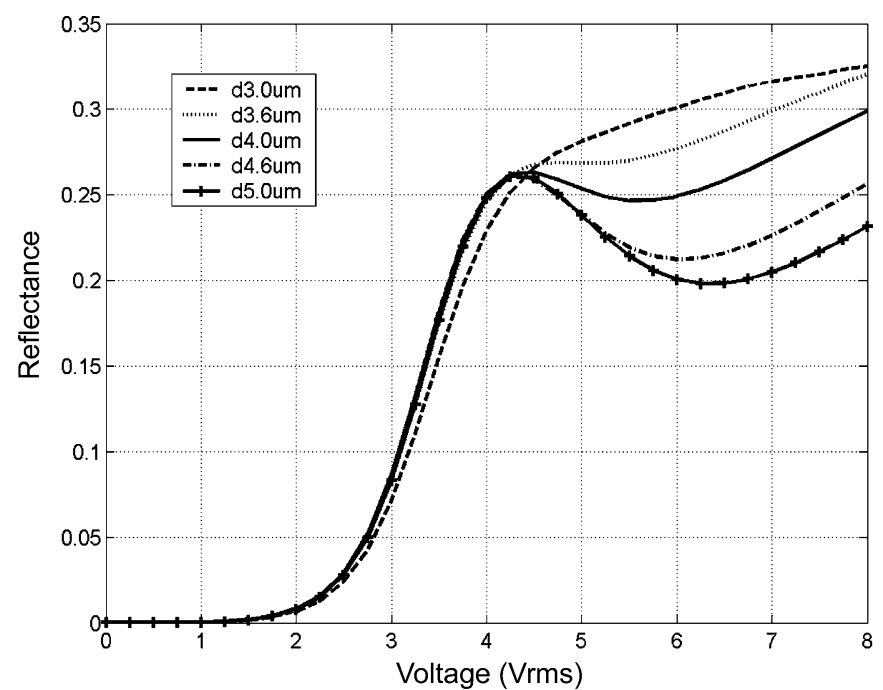

(b)

Fig. 3. (a) VT and (b) VR curves under different cell gaps at $\lambda=550 \mathrm{~nm}$.

partially switched which contributes less to the phase retardation [19]. This difference helps to balance the phase retardation for the T- and R-modes because the light traverses the LC layer twice in the R-mode, but only once in the T-mode.

\section{B. VT and VR Curves at Different Cell Gaps}

Fig. 3 shows the voltage-dependent transmittance (VT) and reflectance (VR) curves at $\lambda=550 \mathrm{~nm}$ under different cell gaps. In our simulations, the maximum transmittance under a pair of polarizers is $37 \%$. In the T-mode, the peak transmittance increases as the cell gap increases from $d=3 \mu \mathrm{m}$ and reaches a maximum $(\sim 33 \%)$ at $d=4 \mu \mathrm{m}$ and $\sim 4.75 V_{\text {rms }}$. Keep on increasing the cell gap leads to a decreased peak transmittance, but its corresponding driving voltage at the maximum transmittance is reduced from $5 V_{\mathrm{rms}}$ for $d=3 \mu \mathrm{m}$ to $4.5 V_{\mathrm{rms}}$ for $\mathrm{d}=5 \mu \mathrm{m}$. The peak transmittance variation shown in Fig. 3(a) originates from the nonuniform LC directors distribution induced by the nonuniform fringing fields.

In the R-mode, the VR curve reaches its first maximum at $\sim 4.3 V_{\text {rms }}$ and then oscillates slightly depending on the cell

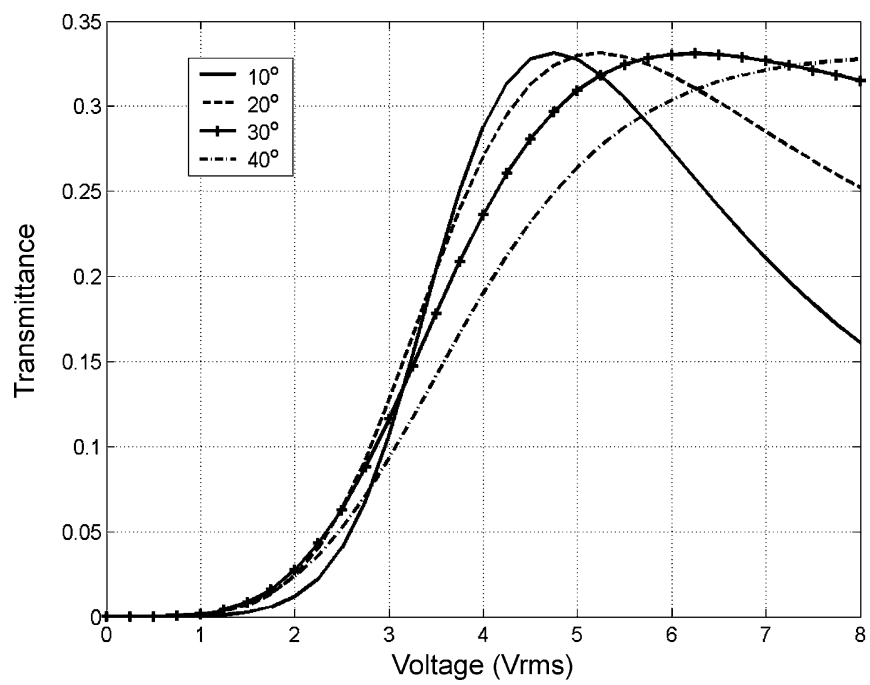

(a)

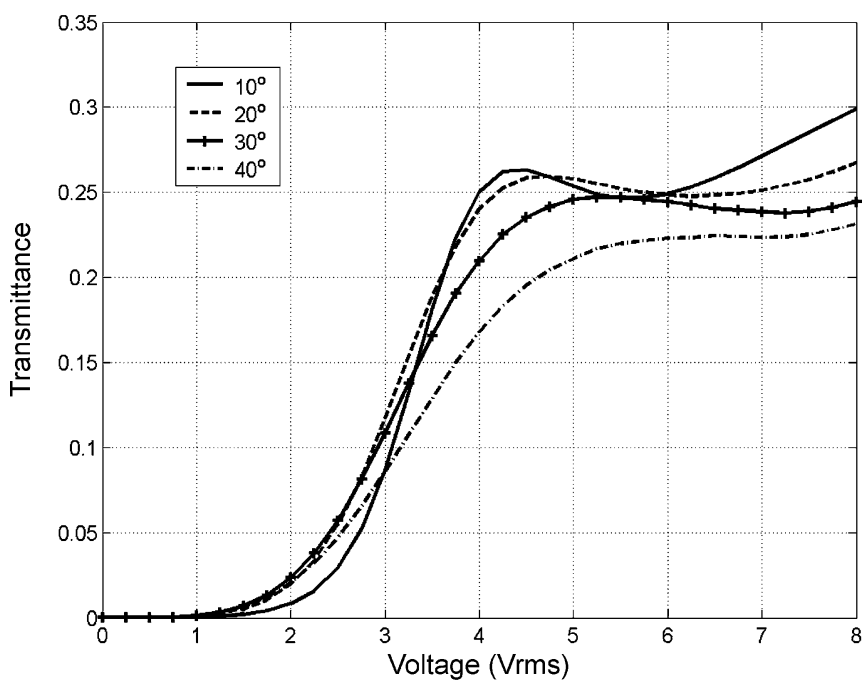

(b)

Fig. 4. (a) VT and (b) VR curves under different rubbing angles at $d=4 \mu \mathrm{m}$ and $\lambda=550 \mathrm{~nm}$.

gap. This abnormal behavior results from two opposite domains induced by the fringing fields which not only tilt but also twist the LC directors. A similar phenomenon is reported in [9]. Different from T-mode, the reflectance of the R-mode decreases with the increasing cell gap, while the corresponding driving voltage at the first peak in the curves is lowered from above 8 $V_{\text {rms }}$ at $d=3 \mu \mathrm{m}$ to $4.5 V_{\text {rms }}$ at $d=5 \mu \mathrm{m}$.

\section{VT and VR Curves at Different Rubbing Angles}

Fig. 4 shows the rubbing angle effect on the VT and VR curves for the $4-\mu \mathrm{m}$ IPS cell at $\lambda=550 \mathrm{~nm}$. For the T-mode, the threshold voltage decreases but the on-state voltage increases as the rubbing angle increases. For a larger rubbing angle, the LC directors can be reoriented more easily leading to a decreased threshold voltage. However, the total phase retardation is reduced so that the on-state voltage is increased. Similar situation occurs for the R-mode [20]. From Fig. 4, the threshold voltage is decreased from $\sim 1.4 V_{\text {rms }}$ at $\phi=10^{\circ}$ to $\sim 1.0 V_{\text {rms }}$ at $\phi=40^{\circ}$ for both T- and R-modes. 


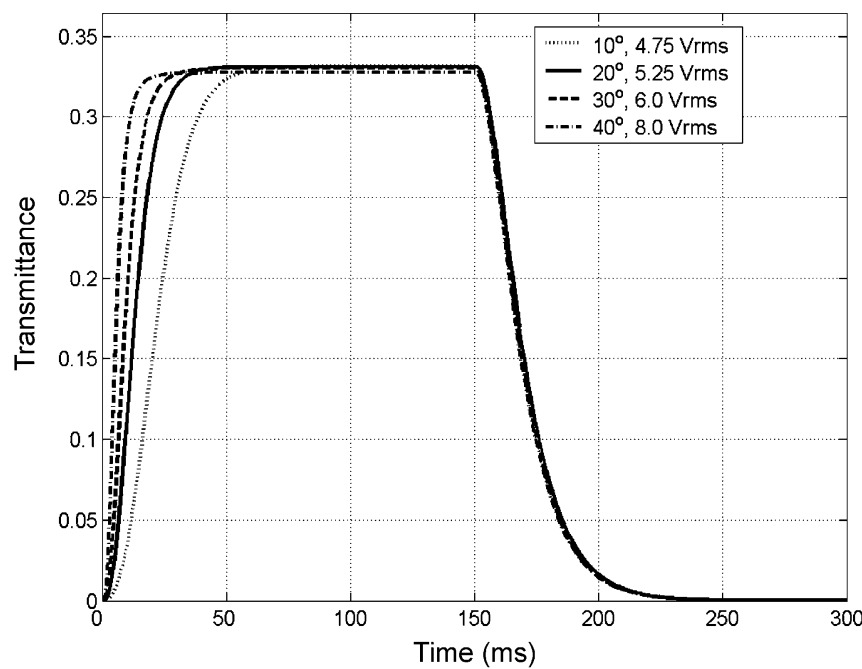

(a)

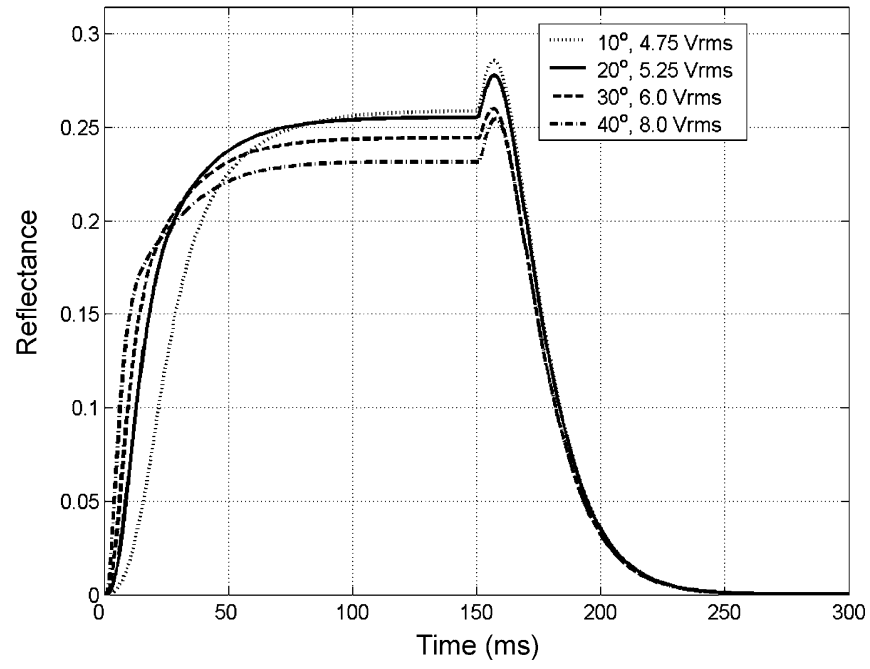

(b)

Fig. 5. (a) Tt curves of T-mode and (b) Rt curves of R-mode with different rubbing angles.

\section{Response Time at Different Rubbing Angles}

Fig. 5 plots the time-dependent transmittance (Tt) curves of the T-mode and R-mode under different rubbing angles for the 4- $\mu \mathrm{m}$ IPS cell at $\lambda=550 \mathrm{~nm}$. The applied voltage is chosen at its respective maximum transmittance/reflectance point. The response time is shortened as the rubbing angle increases for both $\mathrm{T}$ - and R-modes. The improved response time mainly results from the rising period. Specifically, the rise time is shortened from $28.9 \mathrm{~ms}$ at $\phi=10^{\circ}$ to $8.4 \mathrm{~ms}$ at $\phi=40^{\circ}$ for the T-mode, and from $43.9 \mathrm{~ms}$ at $\phi=10^{\circ}$ to $32.6 \mathrm{~ms}$ at $\phi=40^{\circ}$ for the R-mode. The optical rise time is defined from $10 \%$ to $90 \%$ transmission change in the rising period. Although a large rubbing angle helps to reduce rise time, its tradeoff is the increased driving voltage. The longer rise time in the R-mode is due to the weaker effective electric field above the R-regions to switch the LCs, which arises from the parabolic transverse electric field between the neighboring electrodes. In addition, there is a small transient optical bounce in the decay period of the R-mode under different rubbing angles. It is attributed to the

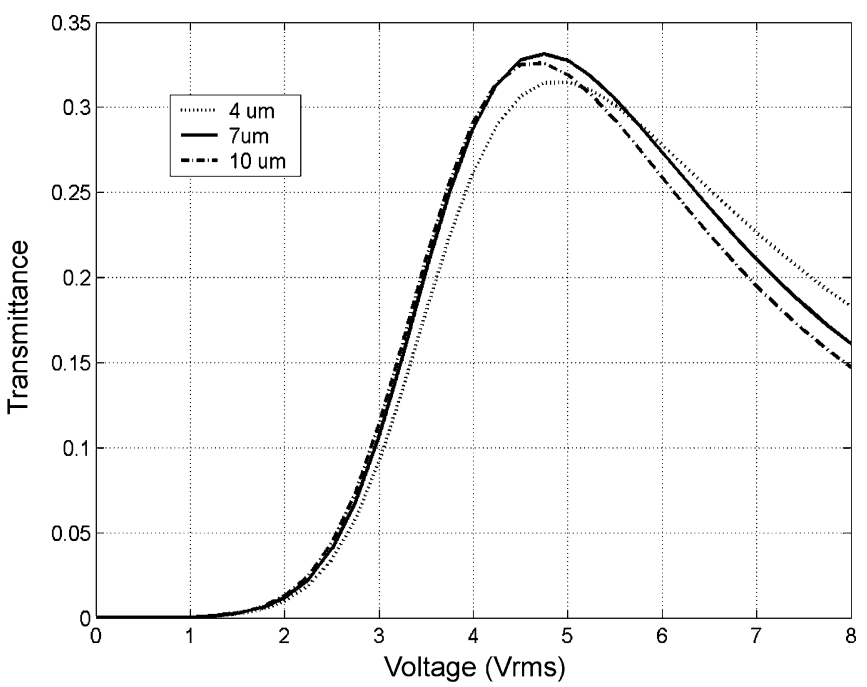

(a)

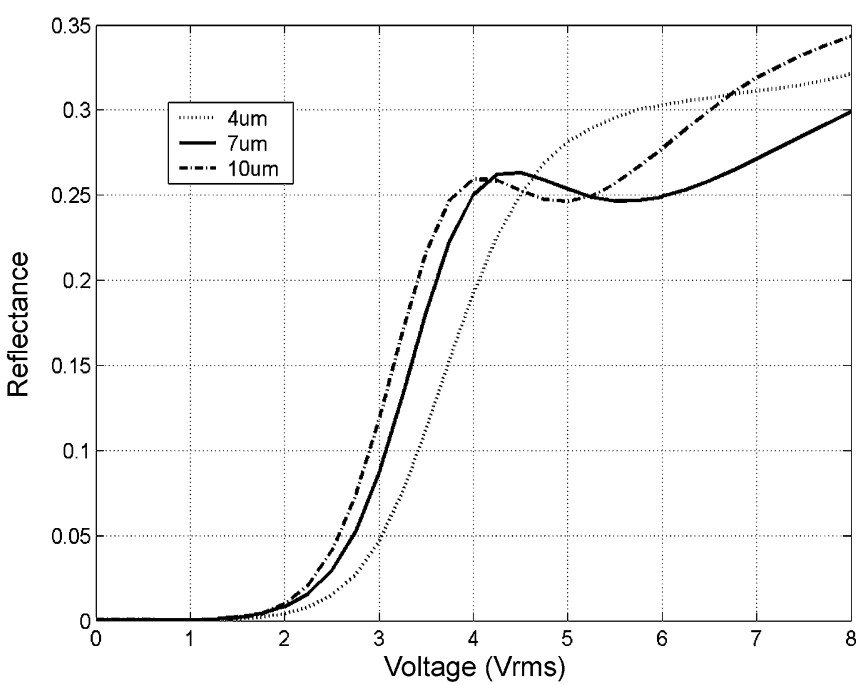

(b)

Fig. 6. (a) VT and (b) VR curves at different reflector widths.

complex director distributions in the R-part as shown in Fig. 2, which involves both twist and splay effects. Therefore, when the applied voltage is released the LC directors relax back with the variation of LC azimuthal and tilt angles simultaneously, which results in the transient optical bounce.

\section{E. Effect of Reflector Width}

In this section, we vary the reflector width to optimize the VT and VR curves. Our goal is to improve the reflectance by optimizing the reflector width in order to tune the effective phase retardation over the R-mode. As Fig. 6 shows, changing the reflector width from 4 to $10 \mu \mathrm{m}$ does not cause significant change to the T-mode under the conditions at $\phi=10^{\circ}, d=4 \mu \mathrm{m}$ and $\lambda=550 \mathrm{~nm}$. By contrast, the R-mode is evidently influenced by the variation of reflector width. At $4 \mu \mathrm{m}$ reflector width, the R-mode has $\sim 28.5 \%$ reflectance at $V=5 V_{\text {rms }}$ which is comparable to the transmittance of the T-mode. With the increased reflector width, the reflectance of the R-mode exhibits a saddle point. The first peak occurs at a lower voltage, but its maximum reflectance is reduced slightly. 


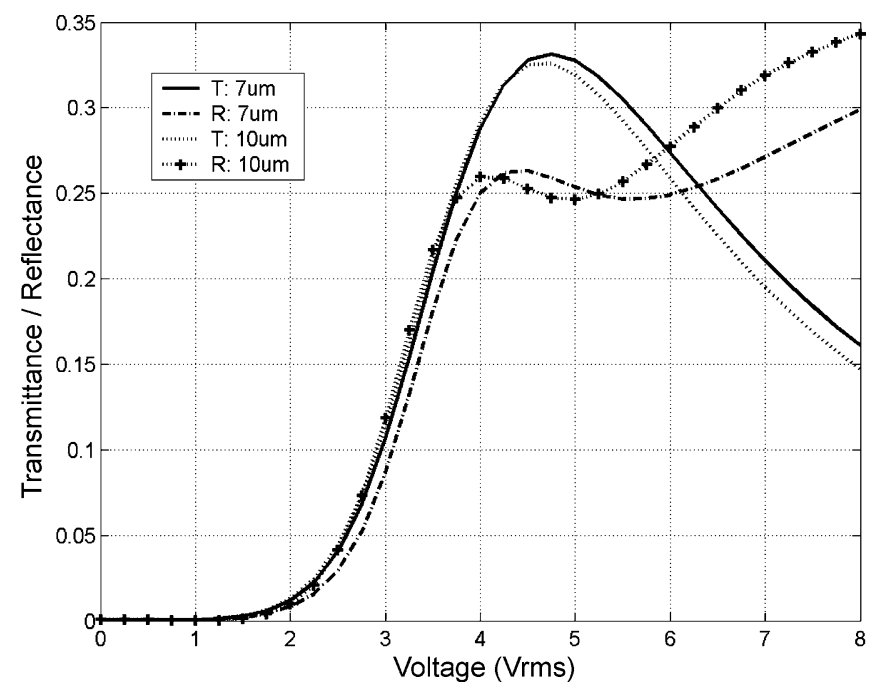

Fig. 7. Gamma curve comparison of the T- and R-modes under two different reflector widths.

Fig. 7 compares the VT/VR curves with different reflector widths for the $4-\mu \mathrm{m}$ IPS cell at $\lambda=550 \mathrm{~nm}$. For the $7-\mu \mathrm{m}$ reflector width, the gamma curves of the T- and R-modes do not overlap very well. As the reflector width is increased to $10 \mu \mathrm{m}$, the VT and VR curves match quite well when the applied voltage is below $4 V_{\text {rms. }}$. Therefore, we can vary the reflector width to match the reflectance curve of the R-mode to the transmittance curve of the $\mathrm{T}$-mode for realizing a single gamma and single TFT transflective IPS-LCD. Between 4.0 and $4.5 V_{\text {rms }}$, the R-mode is basically saturated and its gamma curve departs from that of T-mode.

\section{CONCLUSION}

A transflective IPS-LCD with separate electrodes and reflectors is proposed and its electro-optical properties are simulated. The rubbing angle is found to greatly influence the performance of the transflective IPS-LCD. As the rubbing angle increases, the threshold voltage is reduced and the rise time shortened. In the meantime, the T-mode can still keep the same maximum transmittance even though the reflectance in the R-mode is reduced. The reflector width plays an important role to improve the reflectance curve of the R-mode. Our results indicate that it is possible to realize a high quality single cell gap, single gamma curve and single TFT transflective IPS-LCD by optimizing the reflectance curve of the R-mode to better fit the transmittance curve of the T-mode.

\section{REFERENCES}

[1] S. T. Wu and C. S. Wu, "Mixed-mode twisted nematic liquid crystal cells for reflective displays," Appl. Phys. Lett., vol. 68, pp. 1455-1457, Mar. 1996.

[2] M. Shibazaki, Y. Ukawa, S. Takahashi, Y. Iefuji, and T. Nakagawa, "Transflective LCD with low driving voltage and wide viewing angle," in SID Tech. Dig., 2003, vol. 34, pp. 90-93.

[3] C. L. Yang, I. A. Yao, W. Y. Ling, P. F. Wang, C. J. Chen, J. P. Pang, R. J. Xiang, and S. H. Chen, "A Transflective OCB LCD with wide viewing angle and fast response," in SID Tech. Dig., 2005, vol. 36, pp. 1876-1879.
[4] J. Y. Kim, Y. W. Lim, and S. D. Lee, "Brightness-enhanced transflective liquid crystal display having single-cell gap in vertically aligned configuration,” Jpn. J. Appl. Phys., vol. 45, pp. 810-812, Feb. 2006.

[5] S. H. Lee, T. H. Yoon, and J. C. Kim, "Transflective liquid-crystal display using low-twisted vertically aligned mode," Opt. Lett., vol. 31, pp. 2196-2198, Jul. 2006.

[6] C. L. Yang, "Electro-optics of a transflective liquid crystal display with hybrid-aligned liquid crystal texture," Jpn. J. Appl. Phys., vol. 43, pp. 4273-4275, Jul. 2004.

[7] J. H. Song and S. H. Lee, "A single gap transflective display using in-plane switching mode," Jpn. J. Appl. Phys., vol. 43, pp. L1130-L1132, Aug. 2004.

[8] K. H. Park, J. C. Kim, and T. H. Yoon, "In-plane-switching liquid crystal cell with zigzag electrodes for transflective displays," Jpn. J. Appl. Phys., vol. 43, pp. 7536-7539, Nov. 2004.

[9] G. S. Lee, K. H. Park, J. C. Kim, and T. H. Yoon, "Optimization of electrode structure for single gamma in a transflective IPS LCD," in SID Tech. Digest, 2005, vol. 36, pp. 738-741.

[10] T. B. Jung, J. C. Kim, and S. H. Lee, "Wide-viewing-angle transflective display associated with a fringe-field driven homogeneously aligned nematic liquid crystal display," Jpn. J. Appl. Phys., vol. 42, pp. L464-L467, Mar. 2003.

[11] E. Jeong, M. O. Choi, Y. J. Lim, Y. H. Jeong, H. Y. Kim, S. Y. Kim, and S. H. Lee, "A single gap transflective fringe-field switching display," in SID Tech. Dig., 2006, vol. 37, pp. 810-812.

[12] X. Zhu, Z. Ge, T. X. Wu, and S. T. Wu, "Transflective liquid crystal displays," J. Display Technology, vol. 1, pp. 15-29, Sept. 2005.

[13] N. Sugiura and T. Uchida, "Designing bright reflective full-color LCDs using an optimized reflector," in SID Tech. Dig., 1997, vol. 28, pp. $1011-1014$.

[14] S. Roosendaal, B. van der Zande, A. Nieuwkerk, C. Renders, J. Osenga, C. Doornkamp, E. Peeters, J. Bruinink, J. van Haaren, and S. Takahashi, "Novel high performance transflective LCD with a patterned retarder," in SID Tech. Digest, 2003, vol. 34, pp. 78-81.

[15] R. Lu, S. T. Wu, Z. Ge, Q. Hong, and T. X. Wu, "Bending angle effects on the multi-domain in-plane-switching liquid crystal displays," J. Display Technol., vol. 1, no. 2, pp. 207-216, Dec. 2005.

[16] A. Lien, "A detailed derivation of extended Jones matrix representation for twisted nematic liquid crystal displays," Liq. Cryst., vol. 22, pp. 171-175, Feb. 1997.

[17] C. L. Kuo, C. K. Wei, S. T. Wu, and C. S. Wu, "Reflective direct-view display using a mixed-mode twisted cell," Jpn. J. Appl. Phys., vol. 36, pp. 1077-1080, Mar. 1997.

[18] Z. Ge, T. X. Wu, X. Y. Zhu, and S. T. Wu, "Reflective liquid crystal displays with asymmetric incidence and exit angles," J. Opt. Soc. Amer. A., vol. 22, pp. 966-977, May 2005.

[19] S. T. Wu and D. K. Yang, Reflective Liquid Crystal Displays. New York: Wiley, 2001.

[20] Y. Sun, Z. Zhang, H. Ma, X. Zhu, and S. T. Wu, "Optimal rubbing angle for reflective in-plane switching liquid crystal displays," Appl. Phys. Lett., vol. 81, pp. 4907-4909, Dec. 2002.

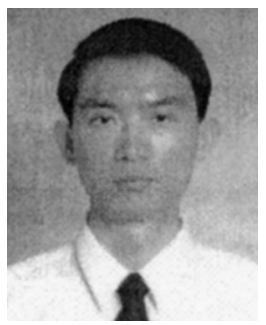

Ruibo Lu received the Ph.D. degree in optics from Department of Physics, Fudan University, Shanghai, China, in 1998, and M.S. degree in applied physics from Department of Physics, East China University of Science and Technology, Shanghai, China, in 1995. His research work in Ph.D. focused on liquid crystal alignment and ferroelectric liquid crystal devices for display and advanced optical applications.

He was a faculty member in the Department of Physics, and later in the Department of Optical Science and Engineering, Fudan University, Shanghai, China, from 1998 to 2001. He was an optical engineer in Lightwaves2020 Inc., San Jose, CA, from 2001 to 2002. Since then, he joined the School of Optics/CREOL (now as College of Optics and Photonics), University of Central Florida, Orlando, as a research scientist. His research interests include liquid crystal display technology, wide viewing angle for liquid crystal TVs, liquid crystal components for optical communications and optical imaging using liquid crystal medium. 


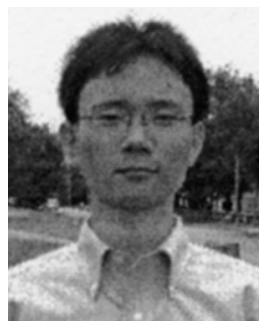

Zhibing Ge received the B.S. and M.S. degrees in electrical engineering from Zhejiang University, China, in 2002, and 2004, respectively. He is currently continuing his $\mathrm{Ph}$. D. study in the area of liquid crystal display modeling and application.

$\mathrm{He}$ joined the Electrical and Computer Engineering Department, University of Central Florida, Orlando, as a graduate student in 2002, and his current research interests include transflective liquid crystal displays and numerical methods in modeling of liquid crystal devices.

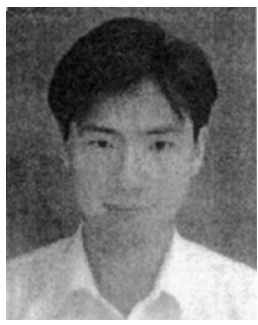

Qi Hong received B.S. degree from the Nanjing University of Aeronautics and Astronautics, Nanjing, China, in 1992, and the M.S.E.E. degree from the University of Central Florida, Orlando, in 2002, where he is currently pursuing the Ph.D. degree in the electrical engineering.

$\mathrm{He}$ was a Design Engineer at the Xiaxin Electronics Company Ltd., Xiamen, China, from 1992 to 2000 . His doctoral research topics include liquid crystal device modeling, wide viewing angle and fast response liquid crystal display.

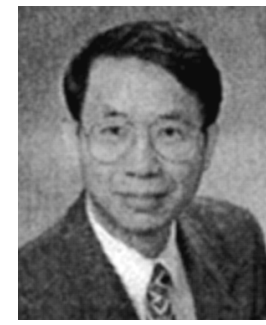

Shin-Tson Wu (M'98-SM'99-F'04) received the B.S. degree in physics from National Taiwan University, and the Ph.D. degree from the University of Southern California, Los Angeles.

$\mathrm{He}$ is a PREP professor at College of Optics and Photonics, University of Central Florida (UCF). His studies at UCF concentrate in foveated imaging, bio-photonics, optical communications, liquid crystal displays, and liquid crystal materials. Prior to joining UCF in 2001, he worked at Hughes Research Laboratories, Malibu, CA, for 18 years. He has co-authored 4 books: Fundamentals of Liquid Crystal Devices (Wiley, 2006, with D. K. Yang); Introduction to Microdisplays (Wiley, 2006, with D. Armitage and I. Underwood) Reflective Liquid Crystal Displays" (Wiley, 2001, with D. K. Yang) and Optics and Nonlinear Optics of Liquid Crystals (World Scientific, 1993, with L. C. Khoo), 5 book chapters, and over 350 journal papers. He has more than 55 issued and pending patents. Several of his patents have been implemented in display and photonic devices.

Dr. Wu is a Fellow of the Society of Information Display (SID) and Optical Society of America (OSA). 